\title{
Comparative Study between Loop Saphenous Vein and Prosthetic Thigh Vascular Access Graft for Haemodialysis
}

\author{
Mohamed Abdel Hamid Abdel Rahman, Ashraf Mohamed Ewida, Ayaman Abd El-Hamid Farag* \\ *Department of Vascular Surgery, Faculty of Medicine, Al-Azhar University \\ *Corresponding author: Ayaman Abd El-Hamid Farag; E-Mail: dr_aymanelsamalosy@yahoo.com;
}

\section{ABSTRACT}

Phone: 02-0111225124 - 01553011983

\begin{abstract}
Background: renal disease is an international problem with a high mortality and morbidity especially in the lowincome countries. Haemodialysis can help patients, but with multiple complications and challenges associated with the procedure. One of these challenges is exhausted veins in the upper limb.

Aim of the work: this study aimed to compare using of great saphenous vein bridge fistula versus prosthetic vascular graft, for creating bridge between superficial femoral artery and vein, for the purpose of regular haemodialysis.

Patients and Methods: 40 chronic renal failure patients were included. Patients were subjected to full history, clinical examination, investigations and Doppler/duplex scanning for both arterial and venous systems. Follow up was done immediately at the first postoperative day then at one week, 3 weeks, 3 months, 6 months, 9 months and after one year as regards patency, functioning of the graft as well as complications.

Results: early death within 30 days of surgery did not occur. Thrombosis was more frequent in PTFE AV graft loop group while all insufficient graft flow $(<200 \mathrm{~mL} /$ minute) cases were in SVI group. The numbers of puncture site complications were similar; however, bleeding was more frequent in PTFE group $(p=0.011)$. None of the patients showed clinical signs or symptoms of graft infection

Conclusions: autogenous arteriovenous fistula reveals better primary patency rate than that of prosthetic arteriovenous graft with statistically significant results $(\mathrm{p}=0.04)$. Autogenous arteriovenous fistula showed statistically better secondary patency rate $(\mathrm{p}=0.001)$.
\end{abstract}

Keywords: Renal failure, Haemodialysis, Graft, Fistula.

\section{INTRODUCTION}

In the light of the ever increasing number of patients with end-stage renal disease, the ageing dialysis population and their prolonged longevity, surgeons are increasingly encountered with difficult access problems, such as exhausted upper extremity access sites and central venous outflow obstruction resulting from previous catheterization ${ }^{(1)}$.

In Egypt, we have a good opportunity to reduce the incidence of kidney failure (chronic and acute) substantially by appropriately chosen models. This is because many of the causes of renal failure are eminently preventable. In fact, a rough estimate is that these programs, if successful, can reduce the incidence by as much as $40 \%{ }^{(2)}$.

The constant evolution of angioaccess techniques in the presence of such circumstances has led to the development of arteriovenous access at different anatomic sites, such as the lower extremity. Femoral prosthetic arteriovenous access placed either in the upper thigh or in the mid-thigh in a loop configuration, as well as autogenous arteriovenous access using the transposed superficial femoral vein, have evolved as alternative access procedures in patients with difficult access problems ${ }^{(3)}$.

There are no opposition on the fact that ideal haemodialysis access should be durable and has low risk of infection and frequency for revision operation to maintain patency. It is true that there are a few researches with analytical results saying there is no difference in patency between autogenous arteriovenous fistula and prosthetic arteriovenous graft
(4). However, according to numerous research results, autogenous arteriovenous fistula showed better results than prosthetic arteriovenous graft ${ }^{(5)}$.

There are various studies comparing the PTFE grafts with biological grafts concerning the infection, patency rate, complications etc. Huber et al. ${ }^{(6)}$ recommend native AVFs as the first step of treatment and in other circumstances offer autologous solutions as possible primary and secondary patency, lower complication rate and cost when compared with PTFE grafts.

\section{AIM OF THE WORK}

The aim of this work is to compare using of great saphenous vein bridge fistula as a venous auto-graft, versus prosthetic vascular graft, for creating loop A-V fistula or A-V graft in the thigh (bridge between superficial femoral artery and vein), in patients with chronic renal failure with exhausted upper extremity accesses, for the purpose of regular haemodialysis, as regards patency (primary and secondary), function criteria (fistula thrill and murmur) and complications.

\section{PATIENTS AND METHODS}

This study was conducted on forty patients with end stage renal disease (13 males and 27 females). 15 patients had diabetes mellitus and 11 patients had hypertension. Patient were divided randomly into two equal groups, autologous saphenous vein was interposed between superficial femoral artery and vein in 20 patients (Group 1). PTFE synthetic graft was interposed between superficial femoral artery and vein 
also in 20 patients (Group 2). The preoperative characteristics were similar in both groups.

- Inclusion criteria: Chronic renal failure patients in need for haemodialysis access in the form of AVF or AV grafts with exhausted access in both Upper limbs.

- Exclusion criteria: Patients with groin or thigh infection, patients with critical lower limb ischemia, patients with occluded femoral artery, patients with occluded deep veins, chronic renal failure patients with any other functioning access in the upper limb, CRF patients with heart failure or $\mathrm{EF}>30 \%$, ABI less than 0.9 .

\section{Ethical approval Written informed consent:}

An approval of the study was obtained from Al- Azhar University Academic and Ethical Committee. Every patient signed an informed written consent for acceptance of the operation.

\section{Methods:}

\section{Patients were subjected to the following:}

1- Full history.

2- Full clinical examination.

3- General examination

4- Local examination to the upper and lower limbs.

5- Chest X-ray.

6- ECG.

7- Echocardiography.

8- Measurement of Ankle brachial pressure index.

9- Duplex Ultrasound for the venous and arterial systems in both lower limbs.

10- CT angiography in patients with claudication pain.

Data were collected regarding age, gender, diabetes mellitus, hypertension, ischaemic heart disease, cerebrovascular accident, dyslipidemia and full details of duplex scanning.

\section{Pre-operative preparations:}

Doppler/duplex scan was done for all patients. The duplex scan protocol included visualization of both arterial and venous systems as well as examination of previous occluded vessels in the upper limb due to previous multiple operations or obliterated or unsuitable veins.

\section{Surgical technique:}

Patients were operated under general or local anaesthesia with mild intravenous sedation. A 15-20 centimeters length saphenous vein graft was harvested proximally.

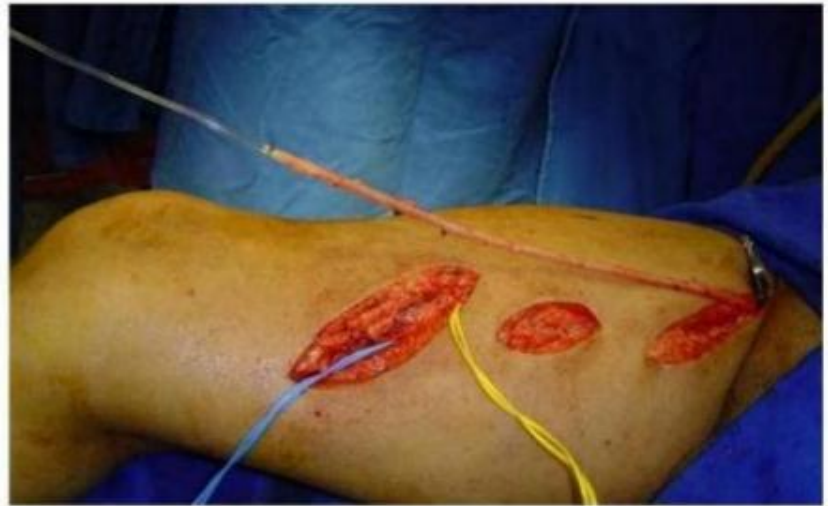

Figure (1): Harvesting great saphenous vein prior to anastomosis with superficial femoral artery.

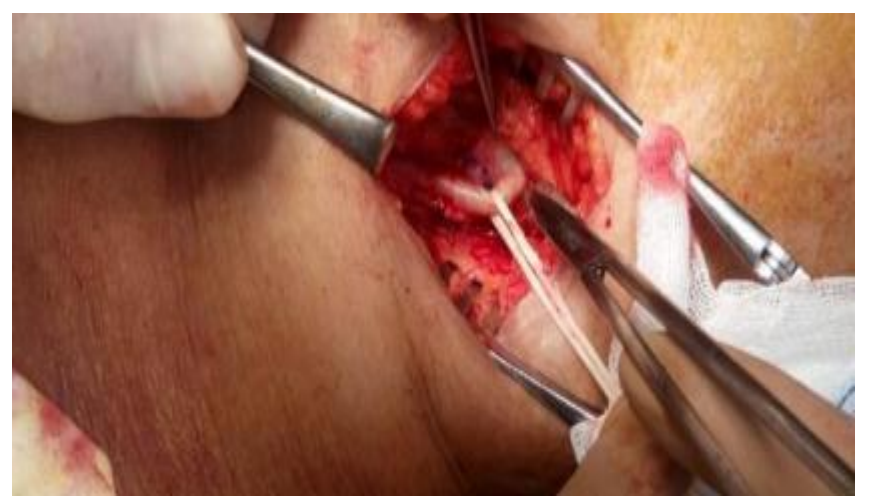

Figure (2): Exposure of superficial femoral artery.

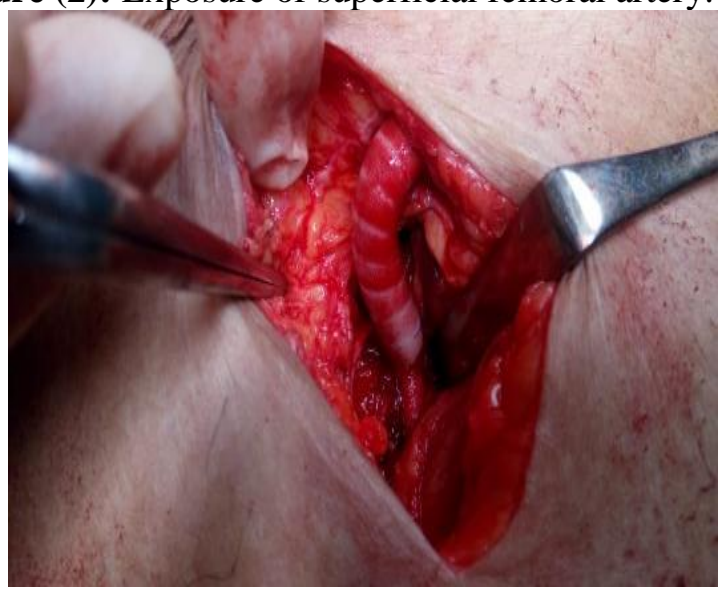

Figure (3): PTFE proximal anastomosis

Superficial femoral artery was found then via a groin incision. A straight tunnel was created in the subcutaneous tissue between previously determined arterial and venous anastomotic site. Following the intravenous heparin administration, autologous saphenous vein (group 1) or polytetrafluoroethylene (PTFE) graft (group 2) was preserved and both the artery and vein were clamped. 


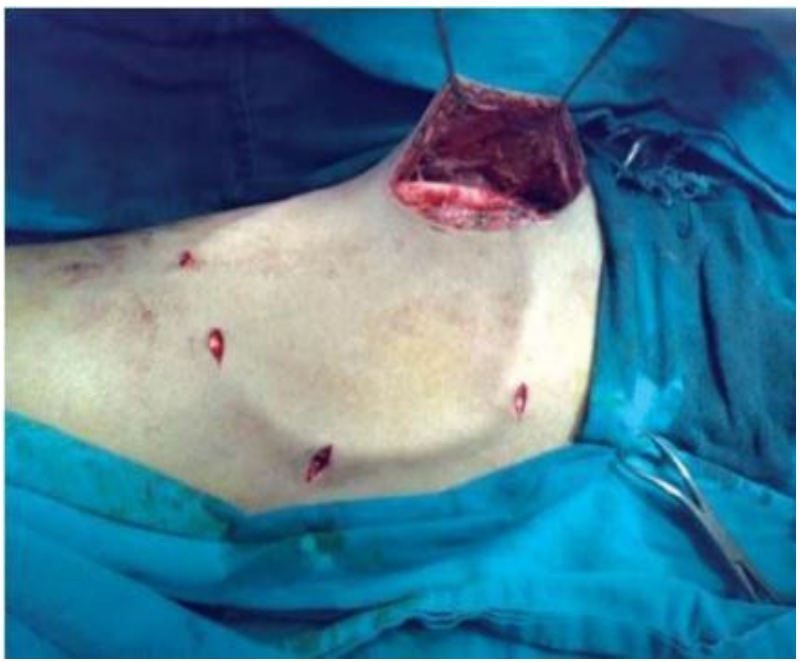

Figure (4): PTFE graft from superficial femoral artery to superficial femoral vein

Arteriotomy for the superficial femoral artery was performed. After completion of the arterial anastomosis, the graft was passed through the tunnel and the fistula tract was carefully checked for any kink or outer compression. The distal (vein) anastomosis was created after performing the venotomy. Venous anastomosis was constituted in manner of patch plasty of the graft to prevent further stenosis of the fistula which was commonly observed in this anastomotic site.

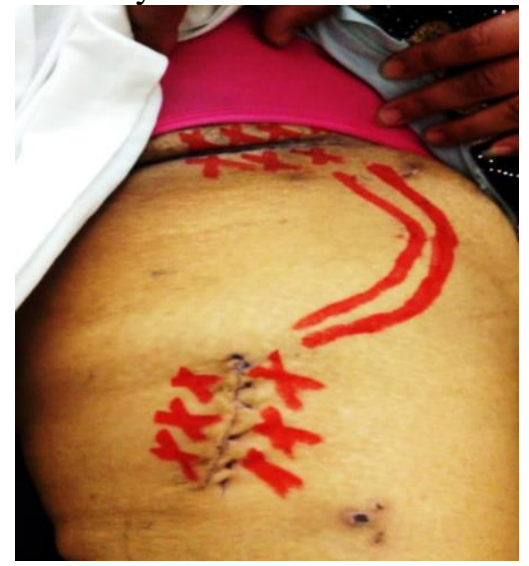

Figure (5): Post-operative marking of the site of arteriovenous fistula to avoid complications.

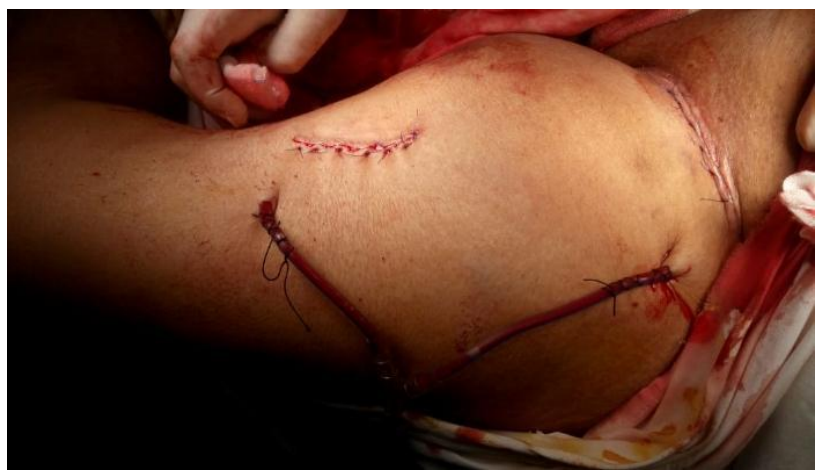

Figure (6): Drain insertion postoperatively

\section{Follow up of patients:}

Patients were evaluated in routine control periods. Follow up immediately at the first postoperative day then at one week, 3 weeks, 3 months, 6 months, 9 months and after one year as regards patency, functioning of the graft as well as complications.

\section{Statistical analysis:}

Recorded data were analyzed using the statistical package for social sciences, version 20.0 (SPSS Inc., Chicago, Illinois, USA). Quantitative data were expressed as mean \pm standard deviation (SD). Qualitative data were expressed as frequency and percentage.

\section{The following tests were done:}

- Independent-samples t-test of significance was used when comparing between two means.

- Chi-square $\left(\mathrm{x}^{2}\right)$ test of significance was used in order to compare proportions between two qualitative parameters.

- The confidence interval was set to $95 \%$ and the margin of error accepted was set to $5 \%$. The p-value was considered significant as the following:

- Probability (P-value)

- P-value < 0.05 was considered significant.

- P-value < 0.001 was considered as highly significant. - P-value > 0.05 was considered insignificant.

\section{RESULTS}

This prospective cohort study was conducted on 40 patients who underwent either interposition saphenous vein (20 patients in group 1) or PTFE graft (20 patients in group 2) as a bridge access for regular haemodialysis. This study was approved by the local Ethics Committee. All patients gave written informed consents.

Table (1) showed demographic data including age in years, sex and associated comorbidities such as hypertension, diabetes mellitus, tobacco use and dyslipidaemia.

Table (1): Preoperative demographics and comorbidities of the patients in both groups

\begin{tabular}{|l|l|l|l|}
\hline \multicolumn{5}{|c|}{ SVI Group PTFE Group value } \\
\hline & $(\mathrm{n}=20)$ & $(\mathrm{n}=20)$ & \\
\hline Age, years & $55.6 \pm 12$ & $57.1 \pm 16$ & 0.87 \\
\hline Male, n \% & $7(35 \%)$ & $6(30 \%)$ & 0.84 \\
\hline Female, $\mathbf{n} \%$ & $13(65 \%)$ & $14(70 \%)$ & 0.7 \\
\hline Associated comorbidities & \multicolumn{3}{|l|}{} \\
\hline Hypertension, n \% & $12(60 \%)$ & $14(70 \%)$ & 0.65 \\
\hline Diabetes mellitus, n \% & $7(35 \%)$ & $8(40 \%)$ & 0.87 \\
\hline Tobacco use, n \% & $4(20 \%)$ & $5(25 \%)$ & 0.79 \\
\hline Hyperlipidemia, n \% & $6(30 \%)$ & $5(25 \%)$ & 0.80 \\
\hline
\end{tabular}

Preoperative mean transverse diameter of the saphenous vein was $3.9 \mathrm{~mm}$ proximally and $3.6 \mathrm{~mm}$ distally in the study group (1). Patients were evaluated in routine control periods. Follow up of patients was immediately at the first postoperative day then at one 
week, 3 weeks, 3 months, 6 months, 9 months and after one year.

\section{Primary patency rate, patency period, and related} factors

Primary patency rates in 3 weeks, 3 months, 6 months, 9 months and one year period were $90 \%, 80 \%, 65 \%$, $60 \%$ and $50 \%$ with autogenous arteriovenous fistula and $65 \%, 50 \%, 30 \%, 25 \%$ and $20 \%$ with prosthetic arteriovenous graft (Figure 7).

\section{Primary Patency rates}

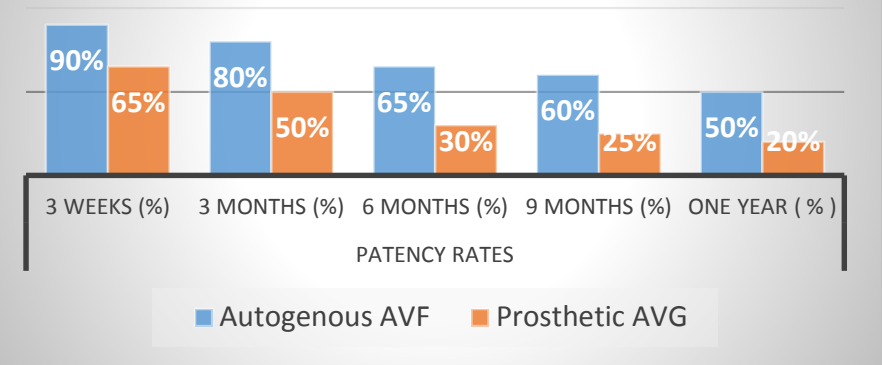

Figure (7): Primary patency rate in both patient groups

Autogenous arteriovenous fistula revealed better primary patency rate than that of prosthetic arteriovenous graft with statistically significant results $(\mathrm{p}=0.04)$ (Fig. 7).

Average primary patency periods were $191.7 \pm 112.7$ days for autogenous arteriovenous fistula and $98.8 \pm$ 79.6 days for prosthetic graft $(\mathrm{p}=0.01)$. It also showed statistically significant results (Figure 8).

\section{Average primary patency periods}

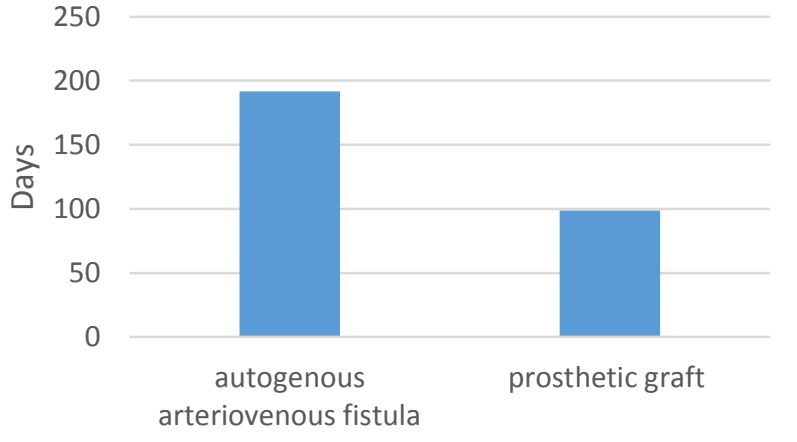

Figure (8): Average primary patency periods Secondary patency rate, patency period, and related factors

Secondary patency rates in 3 weeks, 3 months, 6 months, 9 months and one year period were $95 \%, 90 \%$, $85 \%$, and $65 \%$ with autogenous arteriovenous fistula and $85 \%, 70 \%, 65 \%$, and $20 \%$ with prosthetic arteriovenous graft. This implies that autogenous arteriovenous fistula shows statistically better secondary patency rate $(\mathrm{p}=0.001)$ (Figure 9).

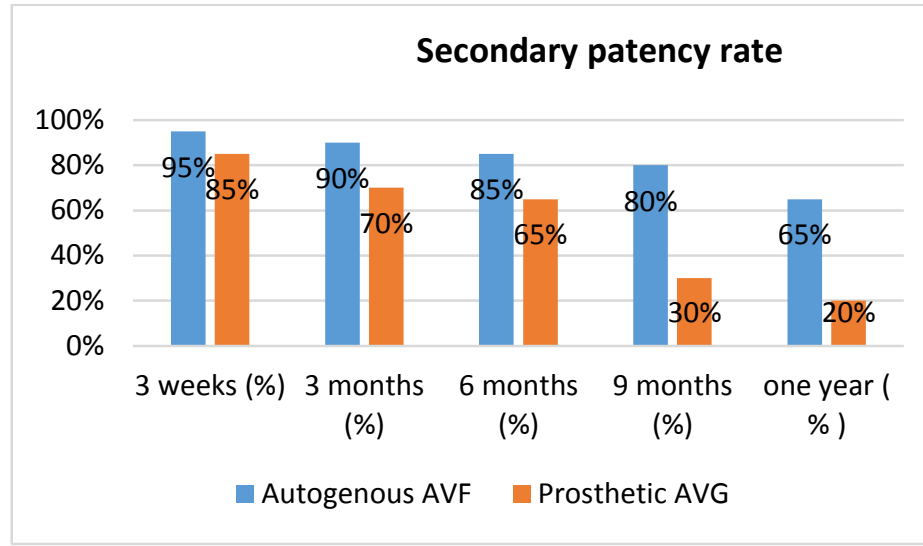

Figure (9): Secondary patency rate in both patient groups

Average secondary patency period was $217.2 \pm 188.7$ days for autogenous arteriovenous fistula and $146.5 \pm$ 102.3 days for prosthetic graft. Autogenous arteriovenous fistula showed better results, but it was not significant statistically $(\mathrm{p}=0.06)$.

Endovascular or surgical revisions for secondary patency in autogenous arteriovenous loop fistula were executed in 10 out of 20 cases $(50.0 \%)$. On the other hand, endovascular or surgical revisions for secondary patency in prosthetic graft were executed in 16 out of 20 cases $(80 \%)$. These results showed that revision occurrence was significantly lower in autogenous arteriovenous loop fistula than prosthetic graft $(\mathrm{p}=0.03)$.

For location of autogenous arteriovenous fistula's stenosis or occlusion, draining vein had the greatest occurrence with 10 cases $(50 \%) .4$ cases occurred at proximal anastomotic site $3 \sim 4 \mathrm{~cm}$ from arteriovenous anastomosis, two cases occurred at central vein, one case occurred at inflow artery, and four cases at other locations.

For methods of revision, balloon angioplasty was used in 2 cases, thrombectomy with graft interposition was used in 2 cases, thrombectomy with patch angioplasty was used in 1 cases, graft interposition was used in 2 cases and simple thrombectomy was used in 3 cases.

\section{Post-operative complications related with both groups}

Early death within 30 days of surgery did not occur. The reasons of failure of the AVFs and related complications were demonstrated in table (2). Thrombosis was more frequent in PTFE AV graft loop group while all insufficient graft flow $(<200$ $\mathrm{mL} /$ minute) cases were in SVI group. The numbers of puncture site complications were similar; however, bleeding was more frequent in PTFE group $(\mathrm{p}=0.011)$. 
None of the patients showed clinical signs or symptoms of graft infection.

One patient suffered from congestive heart failure in SVI group.

Table (2): The complications related to arteriovenous loop fistulae.

\begin{tabular}{|c|c|c|c|}
\hline Complications & $\begin{array}{l}\text { SVI } \\
\text { Group }\end{array}$ & $\begin{array}{l}\text { PTFE } \\
\text { Group }\end{array}$ & \multirow{2}{*}{$\begin{array}{l}\mathbf{p} \\
\text { value }\end{array}$} \\
\hline & $(n=20)$ & $(n=20)$ & \\
\hline Thrombosis, $\mathrm{n} \%$ & $2(10 \%)$ & $9(45 \%)$ & 0.001 \\
\hline Wound infection, $\mathrm{n} \%$ & $1(5 \%)$ & $3(15 \%)$ & 0.05 \\
\hline Insufficient flow, n \% & $3(15 \%)$ & 0 & 0.24 \\
\hline $\mathrm{CHF}, \mathrm{n} \%$ & $1(5 \%)$ & 0 & 1.00 \\
\hline Bleeding, n \% & $3(15 \%)$ & $7(35 \%)$ & 0.011 \\
\hline Haematoma, n \% & $6(30 \%)$ & $2(10 \%)$ & 0.08 \\
\hline Pseudo aneurysm, $\mathrm{n} \%$ & $1(5 \%)$ & 0 & 1.00 \\
\hline
\end{tabular}

Data presented as number of patients and percentages in brackets. Chi-square test or Fisher's exact test were used for data comparison. $\mathrm{P}<0.05$ was considered as statistically significant. CHF - congestive heart failure, PTF polytetrahydroflourene, SVI - saphenous vein interposition.

Thrombosis, infection and bleeding were all significantly higher in PTFE group, while insufficient flow and haematoma were more in saphenous loop group of patients.

\section{DISCUSSION}

According to dialysis outcomes and practice patterns study (DOPPS) (studying international trends of haemodialysis access usage in chronic renal failure patients), frequency of autogenous arteriovenous fistula usage differs from country to country. According to the data after 2005, Japan, Italy, Germany, France, Spain, and England showed $67-91 \%$ of autogenous arteriovenous fistula usage rate whereas United Stated showed the lowest rate of $47 \%{ }^{(7)}$.

Prosthetic vascular access is the other choice when the superficial venous system is inadequate to perform a simple radio-cephalic and brachio-cephalic fistula ${ }^{(8)}$.

Autologous saphenous vein can be preferably chosen as a prosthetic hemodialysis access graft due its higher primary and secondary patency, lower complication rate and cost when compared with PTFE grafts ${ }^{(8)}$.

The incidence of cardiovascular disease was approximately 10 times higher in hemodialysis patients with end-stage renal disease than in the general population ${ }^{(9)}$. The incidence of end-stage renal disease (ESRD) patients is increasing considerably worldwide, and most of the patients start their therapy by hemodialysis (HD). Arteriovenous fistula (AVF) is the best type of vascular access due to its decreased rate of complications followed by arteriovenous graft (AVG) and finally, central venous catheters which are associated with increased mortality and morbidity ${ }^{(\mathbf{1 0})}$.
The difference of autogenous arteriovenous fistula usage rate in each countries can be assumed as the fact that occurrence rates of diabetes, angina pectoris, and peripheral vascular disease are greater in patients from the United States than in patients from Japan or other Europe countries. However, the fact that frequency of autogenous arteriovenous fistula usage in patients from the United States is lower than patients from Europe even after modifying associated other diseases can be implied as the high preference to prosthetic graft among surgeons in US (11).

Ideal haemodialysis access should be durable and has low risk of infection and frequency for revision operation to maintain patency. It is true that there are a few researches with analytical results saying there is no difference in patency between autogenous arteriovenous fistula and prosthetic arteriovenous graft (12, 13). However, according to numerous research results, autogenous arteriovenous loop fistula showed better results than prosthetic arteriovenous loop graft ${ }^{(\mathbf{1 4}}$, 15, and 16). The present study showed that autogenous arteriovenous loop fistula gives better results in frequency of revision operation and primary \& secondary patency rate.

There are various studies comparing the PTFE grafts with biological grafts according to the infection, patency rate, complications etc. Huber et al. (6) recommend native loop AVFs as the first step of treatment and in other circumstances offer autologous solutions as possible primary and secondary patency, lower complication rate and cost when compared to PTFE loop grafts.

According to a large research result from the United States, in 2-year patency rate, primary patency rate of autogenous arteriovenous fistula showed better results with $39.8 \%$ when compared with that of prosthetic graft with $24.6 \%$. However, secondary patency rate difference between autogenous arteriovenous fistula and prosthetic graft was not significant with $64.3 \%$ and $59.5 \%$. In addition, autogenous arteriovenous fistula using venous transposition showed similar secondary patency rate when compared to simple autogenous arteriovenous fistula. It was also shown that female, elders, or patients with previous arteriovenous fistula failure had benefits using the venous transposition fistula (16).

Woo et al. (15) showed in his research about comparison between autogenous arteriovenous fistula using venous transposition and linear shaped prosthetic graft that autogenous fistula was better than prosthetic graft in both primary $(48 \%$ vs. $14 \%)$ and secondary $(57 \%$ vs. $19 \%)$ patency rate at 5-year. In addition, revision frequency for secondary patency was significantly lower in autogenous arteriovenous fistula. They insisted that autogenous arteriovenous fistula should be primarily made when anatomical conditions satisfy with adequate arterial inflow bloodstream and large diameter of vein greater than $2.5 \mathrm{~mm}$. 
Although beginning haemodialysis with a mature autogenous arteriovenous fistula is ideal in all patients without using the catheter, there are numerous obstacles to overcome. The patients must be referred to nephrologist before terminal stage of renal failure, arteriovenous fistula operation must be executed beforehand for maturity and a trained dialysis nurse must successfully execute needling. However, if one of the above conditions is not met, using the catheter is inevitable. In case of the United States, approximately $60-65 \%$ of the patients begin haemodialysis with the catheter ${ }^{(17)}$.

According to Kidney Disease Outcomes Quality Initiative (KDOQI) clinical guideline, autogenous arteriovenous fistula must be made approximately 6 months before the estimated haemodialysis treatment. This is because periodic evaluation for maturation and additional revisions before beginning of haemodialysis are necessary. Average maturation term of autogenous arteriovenous fistula is 2-4 months and that of prosthetic arteriovenous graft is approximately 3-6 weeks ${ }^{(18) .}$

According to KDOQI clinical guideline revised in 2006 , greater than $65 \%$ of the haemodialysis patients are suggested to use autogenous arteriovenous fistula. In order to increase usage frequency of autogenous arteriovenous fistula and achieve successful haemodialysis access, preoperative thorough history investigation, physical examination and ultrasonic vessel mapping are necessary. Using autogenous vessel might be challenging, especially in patients who are diabetic, old, female, or has peripheral vascular disease or severe heart failure. Vein mapping using ultrasound can help deciding the operable site by evaluating the diameter, patency, continuity, and distensibility (19).

Silva et al. ${ }^{(20)}$ reported that non-invasive preoperative test could increase the use of autogenous arteriovenous fistula, reduce postoperative early failure, and improve secondary patency rate.

As a baseline for surgery, the arterial system of the lower limb must be patent, and the venous diameter must be greater than $4 \mathrm{~mm}$ for autogenous fistula proximally. Also, there should be no narrowed or blocked segment, continuity with deep vein must be maintained and no stricture or occlusion in the ipsilateral central vein ${ }^{(20)}$.

Jo et al. ${ }^{(21)}$ reported in their research that there are no difference in patency rate between autogenous loop arteriovenous fistula and prosthetic loop arteriovenous graft. In the present study, however, autogenous loop arteriovenous fistula showed better long-term results both in primary and secondary patency rate. In analysis of risk factors affecting patency rate, diabetes appeared more frequently in prosthetic loop arteriovenous graft (21). However, diabetes alone was not a risk factor of primary and secondary patency rate. In cases where diabetes was followed by ischaemic heart disease, it appeared as a significant risk factor in primary patency rate. Age appeared as a significant risk factor in secondary patency rate (21). PTFE and autologous saphenous vein grafts are commonly used for that purpose. PTFE graft interpositions provide a fast vascular access following the surgery; however, thrombosis and infections may be more frequently seen when compared to autologous saphenous vein ${ }^{(\mathbf{1 4})}$. The definition of the infection of an AVF includes induration, swelling, redness, increase in temperature on AVF tract and drainage from needle or incision site with or without fever and/ or bacteraemia. Any local infection was recorded in both groups in this study ${ }^{\mathbf{( 1 4 2 2} \text {, }}$ 23).

In the present study, infection occurred in one patient $(5 \%)$ in group 1 and 3 patients (15\%) in group 2 (PTFE loop graft). Complications that had no statistical differences between the two groups included insufficient flow, congestive heart failure due to increased flow, haematoma and pseudo-aneurysm. Prolonged bleeding from the needle puncture site was predominantly observed in PTFE group. Local compression or simple suturing over the puncture site was enough to stop bleeding.

In the present study, thrombosis of the graft was a serious complication threatening the patency of the graft and was diagnosed in 9 patients (45\%) in PTFE group and 2 patients $(10 \%)$ in SVI group. Thrombectomy was performed for these patients. The primary and secondary patency of the SVI group was significantly higher in the whole follow up period.

In the present study, bleeding occurred in 3 patients $(15 \%)$ of group 1 and 7 patients $(35 \%)$ of group 2 while pseudo aneurysms occurred in one patient $(5 \%)$ of group 1 and nothing in group 2 .

Native superficial veins were inadequate to perform upper limb AVF in the study group and patients underwent lower limb graft interposition surgery (SVI group and PTFE graft group).

Experience of Silva et al. ${ }^{(20)}$ and Lauvao et al. (24) showed that vein size is the major predictor for a successful fistula. The present study showed the same results

No mortality reported in the present study during patient follow up period.

\section{CONCLUSIONS}

The incidence of ischaemic complications resulting from lower extremity vascular access construction seems to be higher than the reported rates in upper extremity proximal arterio venous accesses. So, we suggest good preoperative screening methods for peripheral arterial disease with a detailed clinical evaluation and duplex ultrasound scanning and/or arteriography when required.

Infection tends to be more common in upper or midthigh and the overall prosthetic than autologous lower extremity AV access. So, we can keep infection rate at low levels by using perioperative prophylactic 
antibiotics and complete aseptic technique at the time of cannulation for haemodialysis.

In this study, autogenous loop arteriovenous fistula showed better results than prosthetic arteriovenous graft in both primary and secondary patency rate. It also showed lower frequency in revisions due to complications such as stricture or thrombosis.

\section{REFERENCES}

1. Gibbons CP (2009): Vascular access in the lower limb. European Journal of Vascular and Endovascular Surgery, 38 (3): 373-374.

2. Barsoum $R$ (2011): A mission in evolution: the International Society of Nephrology in the past 10 years(2001-2010) Kidney International J., 79: 935-943.

3. Tokars JL, Miller ER, Stein G (2002): New national surveillance system for haemodialysis-associated infections: initial results. Am J Infect Control., 30: 288295.

4. Snyder DC, Clericuzio CP, Stringer A et al. (2008): Comparison of outcomes of arteriovenous grafts and fistulas at a single Veterans' Affairs medical center. Am J Surg., 196: 641-6.

5. Perera GB, Mueller MP, Kubaska SM et al. (2004): Superiority of autogenous arteriovenous haemodialysis access: maintenance of function with fewer secon dary interventions. Ann Vasc Surg., 18: 66-73.

6. Huber TS, Ozaki CK, Flynn TC et al. (2002): Prospective validation of an algorithm to maximize native arteriovenous fistulae for chronic haemodialysis access. J Vasc Surg., 36: 452-9.

7. Ethier J, Mendelssohn DC, Elder SJ et al. (2008): Vascular access use and outcomes: an international perspective from the Dialysis Outcomes and Practice Patterns Study. Nephrol Dial Transplant, 23: 3219-26.

8. Uzun A, Diken AI, Yalçinkaya A et al. (2014): Longterm patency of autogenous saphenous veins vs. PTFE interposition graft for prosthetic hemodialysis access. Anadolu Kardiyol Derg., 14 (6): 542-6.

9. Sun Y, Ding W, Wei Q et al. (2012): Dysregulated gene expression of extracellular matrix and adhesion molecules in saphenous vein conduits of hemodialysis patients. J Thorac Cardiovasc Surg., 144 (3): 684-9.

10. Hamdan Z, As'ad N, Sawalmeh O et al. (2019): Vascular access types in hemodialysis patients in palestine and factors affecting their distribution: A cross-sectional study. Saudi J Kidney Dis Transpl., 30 (1): 166-174.

11.Port FK, Pisoni RL, Bommer J et al. (2006): Improving outcomes for dialysis patients in the international Dialysis Outcomes and Practice Patterns Study. Clin J Am Soc Nephrol., 1: 246-55.
12.Snyder DC, Clericuzio CP, Stringer A et al. (2008): Comparison of outcomes of arteriovenous grafts and fistulas at a single Veterans' Affairs medical center. Am J Surg., 196: 641-6.

13. Hodges TC, Fillinger MF, Zwolak RM et al. (1997): Longitudinal comparison of dialysis access methods: Risk factors for failure. J Vasc Surg., 26: 1009- 19.

14. Huber TS, Carter JW, Carter RL et al. (2003): Patency of autogenous and polytetrafluoroethylene upper extremity arteriovenous haemodialysis accesses: a systematic review. J Vasc Surg., 38: 1005-11.

15. Woo K, Doros G, Ng T et al. (2009): Comparison of the efficacy of upper arm transposed arteriovenous fistulae and upper arm prosthetic grafts. J Vasc Surg., 50: 1405-11.

16. Gibson KD, Gillen DL, Caps MT et al. (2001): Vascular access survival and incidence of revisions: a comparison of prosthetic grafts, simple autogenous fistulas, and venous transposition fistulas from the United States Renal Data System Dialysis Morbidity and Mortality Study. J Vasc Surg., 34: 694-700.

17. Allon M, Lockhart ME, Lilly RZ et al. (2011): Effect of preoperative sonographic mapping on vascular access outcomes in haemodialysis patients. Kidney Int., 80 (5): 2013-2020.

18. Brouwer D, McMurray PA, Timothy E et al. (2006): Vascular Access 2006 work Group. Clinical practice guide- lines for vascular access. Am J Kidney Dis., 48: S176- S247.

19.Sidawy AN, Spergel LM, Besarab A et al. (2008): The Society for Vascular Surgery: clinical practice guidelines for the surgical placement and maintenance of arteriovenous haemodialysis access. J Vasc Surg., 48: 225.

20.Silva MB, Hobson RW, Pappas PJ et al. (1998): A strategy for increasing use of autogenous haemodialysis access procedures: impact of preoperative noninvasive evaluation. J Vasc Surg., 27: 302-8.

21.Jo WM, Sohn YS, Rhu SM et al. (2002): Clinical analysis of arteriovenous fistulas for haemodialysis. Korean J Thorac Cardiovasc Surg., 35: 369-74.

22.Lioupis C, Mistry H, Rix T et al. (2011): Comparison among transposed brachiobasilic, brachiobrachial arteriovenous fistulas and Flixene ${ }^{\mathrm{TM}}$ vascular graft. J Vasc Access., 12: 36-44.

23. Bachleda P, Utikal P, Kalinova L et al. (2010): Infectious complications of arteriovenous ePTFE grafts for haemodialysis. Biomed Pap Med Fac Univ Palacky Olomouc Czech Repub., 154: 13-9.

24. Lauvao LS, Ihnat DM, Goshima KR et al. (2009): Vein diameter is the major predictor of fistula maturation. $\mathbf{J}$ Vasc Surg., 49: 1499-504. 